\title{
Forschungsdaten
}

Annette Strauch*

\section{Forschungsdatenmanagement an der Stiftung Universität Hildesheim}

\author{
Praktische Unterstützung für Forschende und Studierende durch die Universitätsbibliothek
}

https://doi.org/10.1515/iwp-2019-2052

Dieser Artikel stellt die Arbeiten ${ }^{1}$ des Forschungsdatenmanagements $(\mathrm{FDM})^{2}$ an der Stiftung Universität Hildesheim $(\mathrm{SUH})^{3}$ vor. Angesiedelt ist dieses institutionelle Angebot für alle Fachbereiche und Institute an der UB Hildesheim in Form einer dauerhaft eingerichteten Beratungsstelle. ${ }^{4}$ Unter Forschungsdaten werden hier alle Daten verstanden, die im Forschungsprozess entstehen, beziehungsweise Ergebnisse sind. Die gegenwärtige Forschung nutzt neue Informations- und Kommunikationstechnologien und ist stark vernetzt. Forschende sollen ohne viel Mehraufwand neben ihrer eigentlichen Forschungsarbeit, Fragen zu einem sinnvollen Umgang mit den Daten, die sie generieren und bearbeiten, beantworten können. Desweiteren haben sich dieErwartungen an eine nachnutzbare Forschung verändert. Es besteht eine breitere Bereitschaft für eine kollaborative Forschungsarbeit über alle Fächergrenzen hinweg.

Deshalb müssen auch die Methodenkompetenzen Forschender erweitert werden. Die forschungsunterstützende Infrastruktur der UB Hildesheim berät vor diesem Hintergrund Forschende $\mathrm{zu}$ allen anfallenden Themen. Dabei orientiert sie sich an den Empfehlungen aus dem LEARN RDM Toolkit. ${ }^{5}$ Im Frühjahr 2018 wurde zunächst

1 Die Aktivitäten des Forschungsdatenmanagement in der UB Hildesheim werden über einen Weblog (https://www.uni-hildesheim.d e/forschungsdaten/) und den Social-Media-Kanal Twitter (@fdmhildesheim) sowie über einen Newsletter angekündigt.

2 https://www.forschungsdaten.org/index.php/Forschungsdatenm anagement [15.9.2019].

3 https://www.uni-hildesheim.de/ [15.09.2019]. https://www.uni-hildesheim.de/bibliothek/ [15.9.2019].

$4 \mathrm{https}$ ://www.dfg.de/foerderung/grundlagen_rahmenbedingun gen/gwp/ [15.9.2019].

5 http://learn-rdm.eu/en/about/ [29.9.2019].

*Kontaktperson: Annette Strauch, M. A., Universitätsbibliothek Hildesheim, Forschungsdatenmanagement, Universitätsplatz 1, 31141 Hildesheim, E-Mail: annette.strauch@uni-hildesheim.de eine allgemeine Informationsseite mit weiterführenden Links zum Forschungsdatenmanagement verfasst. ${ }^{6}$ Den Instituten und Fachbereichen wird empfohlen, sich bei der nationalen und internationalen Etablierung von Forschungsdateninfrastrukturen zu beteiligen. Das Management der Forschungsdaten gehört in den Bereich der ,guten wissenschaftlichen Praxis”, gemäß der „Leitlinien zur Sicherung guter wissenschaftlicher Praxis“ (Kodex), der Deutschen Forschungsgemeinschaft (DFG), ${ }^{7}$ die im Sommer 2019 in Kraft traten. Auch eigene, hochschulinterne Leitlinien für eine institutionelle Forschungsdaten-Politik ${ }^{8}$ für die SUH werden geschrieben und sollen nach Abstimmung in den einzelnen Gremien bald verabschiedet werden. Gemäß der 19. HRK-Mitgliederversammlung (2015) ${ }^{9}$ soll es vor Ort Unterstützung zur Orientierung im Datenmanagement geben. Die Datenkultur wird lokal gestärkt, Infrastrukturen werden aufgebaut und Kompetenzen weiterentwickelt. Die Eckpunkte der Digitalisierungsoffensive für die niedersächsischen Hochschulen $(2017)^{10}$ sowie das Landes-IT-Konzept für Hochschulen in Niedersachsen 2015 bis $2020^{11}$ werden beim Aufbau der Dienstleistungen und technischen Infrastrukturen berücksichtigt.

6 https://www.uni-hildesheim.de/bibliothek/forschen-publizieren/ forschungsdatenmanagement/ [15.9.2019].

7 https://www.dfg.de/foerderung/grundlagen_rahmenbedingun gen/gwp/ [15.9.2019].

8 https://www.forschungsdaten.org/index.php/Data_Policies [15.9.2019].

9 https://www.hrk.de/uploads/tx_szconvention/Empfehlung orschungsdatenmanagement_final_Stand_11.11.2015.pdf[15.9.2019]. 10 https://www.lhk-niedersachsen.de/fileadmin/user_upload/ Eckpunktepapier_f\%C3\%BCr_eine_Digitalisierungsoffensive_der_ nds._Hochschulen_nach_LHK-Beschlussfassung_am_22.05.17_final. pdf [15.9.2019].

11 https://www.lanit-hrz.de/fileadmin/user_upload/Landes-ITKonzept.pdf [15.9.2019]. 


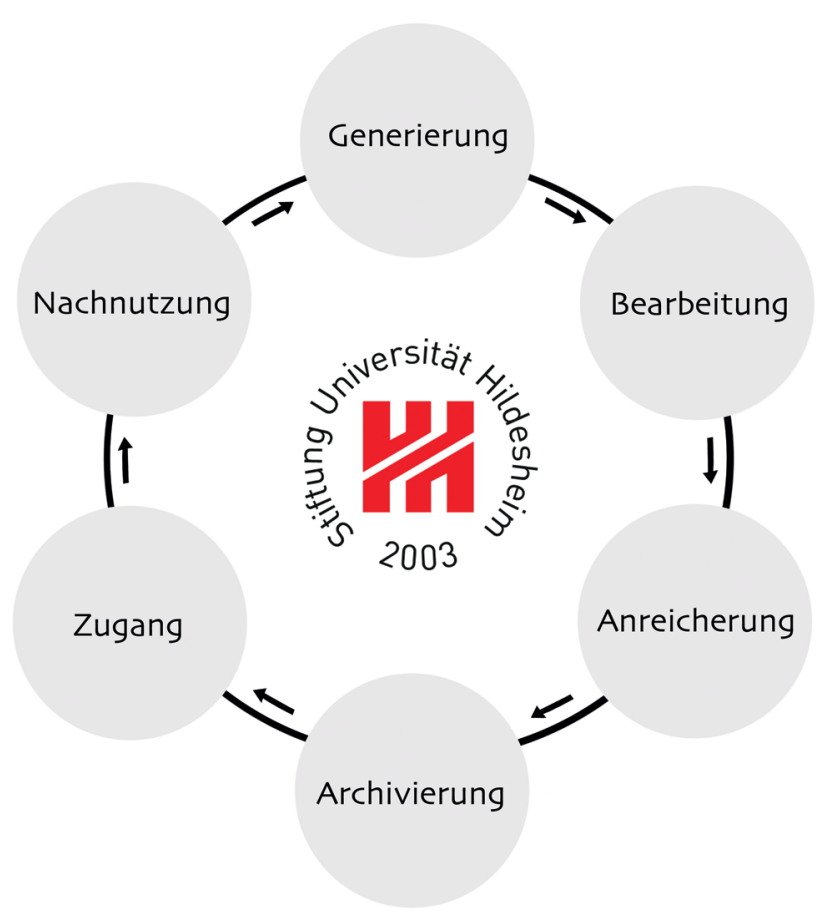

Abbildung 1: „Data Life Cycle“, Stiftung Universität Hildesheim.

Empfohlen wird ein Datenmanagement rund um den „Data Life Cycle” und entlang der Fragen aus den Fragenkatalogen, die in einem Datenmanagementplan (DMP) vorkommen können. Deshalb richten sich die Beratungs- und Dienstleistungsangebote sowie die konkreten Schulungen zunächst immer an diesen beiden Grundpfeilern des Forschungsdatenmanagements aus.

\section{Data Life Cycle}

Die Stationen des Datenlebenszyklus („Data Life Cycle“), dem die UB Hildesheim folgt, werden auf der Webseite der Plattform forschungsdaten.info ${ }^{12}$ näher beschrieben und sind in Abbildung $1 \mathrm{zu}$ sehen (Generierung, Bearbeitung, Anreicherung, Archivierung, Zugang, Nachnutzung).

\section{Datenmanagementplan (DMP)}

Die FDM-Stelle der UB empfiehlt schon vor der Antragstellung eines neuen Forschungsprojekts einen $\mathrm{DMP}^{13} \mathrm{zu}$ er-

12 https://www.forschungsdaten.info/themen/planen-und-struktur ieren/datenlebenszyklus/ [29.9.2019].

$13 \mathrm{https}$ //www.forschungsdaten-bildung.de/datenmanagement plan [29.9.2019]. stellen, damit ein systematischer Umgang mit den Daten ermöglicht und die Beantragung für Fördermittel erleichtert wird. Das Schreiben eines DMP wird durch die UB praktisch unterstützt. Empfohlen wird die Benutzung des Werkzeuges RDMO, ${ }^{14}$ dessen Verwendung in einer persönlichen Beratung erklärt wird. Mit RDMO kann der Umgang mit Forschungsdaten eines universitären Forschungsprojekts strukturiert erfolgen. Forschende berichten, wie sie während der Laufzeit und nach dem Ende eines Projekts mit ihren Forschungsdaten umgehen. Es werden Angaben zum gesamten Projektvorhaben gemacht. Die existierenden Daten werden beschrieben, Datenarten und Formate angegeben und die Datenorgansation sowie die Rechtsthematik behandelt. Die UB Hildesheim gehört der „Research Data Management Organiser“ (RDMO)-Community $^{15}$ an und stellt mittels einer eigenen RDMO Instanz, die im Mai 2018 installiert wurde, eine Webapplikation bereit. ${ }^{16}$ Bei der Zusammenarbeit mit der RDMO-Community lassen die Entwickler zusammen mit den Anwendern von RDMO Anforderungen und Feedback einfließen. Somit wird ein Austausch mit Infrastrukturinitiativen sichergestellt, zum allgemeinen und fachspezifischen Forschungsdatenmanagement.

\section{Rechtsthemen}

Die Schutzwürdigkeit der Daten muss immer geklärt sein. ${ }^{17}$ Fragen, die in diesem Zusammenhang häufig auftauchen, sind: Wer hat welche Rechte an den von mir erzeugten Forschungsdaten? Darf ich meine Forschungsdaten einfach so veröffentlichen? Was sollte ich bei der Nachnutzung von Daten beachten? Wie stelle ich Forschungsdaten Dritten zur Verfügung? Bin ich haftbar, wenn es zu einem Rechtsstreit um von mir veröffentlichte Forschungsdaten kommt? Wer ist für technische Sicherungsmaßnahmen verantwortlich? Bei rechtlichen Themen sind die Fachdisziplin und die Art der Forschungsdaten entscheidend. So entstehen Urheberrechte bei sogenannten persönlichen geistigen Schöpfungen. Daher wird eine schriftliche Zustimmung vor den Veröffentlichungen benötigt. Dazu können personenbezogene Daten anonymisiert werden. Doch manchmal sind Forschungsdaten ethisch problematisch. Solche rechtlichen Themen werden sehr oft an die FDM-Stelle der UB herangetragen. Diese berät zum Beispiel, wie man urheber-

14 https://rdmorganiser.github.io/ [29.9.2019]. 15 https://rdmorganiser.github.io/kooperationen/ [29.9.2019]. $16 \mathrm{https} / / /$ dmp.uni-hildesheim.de/ [29.9.2019]. 17 https://www.forschungsdaten.info/themen/rechte-undpflichten/ [29.9.2019]. 
rechtlich schutzfähige Daten mit einer Lizenz versehen oder wie man sensible Daten in einem fachspezifischen Repositorium speichern kann. Dazu werden Formulierungshilfen für informierte Einwilligungen hochschulintern zur Verfügung gestellt. Die fachspezifischen Bedürfnisse der Forschenden von der Entstehung der Forschungsdaten bis hin zur digitalen Langzeitarchivierunghaben eine große Bandbreite. Daher braucht es hier besonders viel Beratung. Dazu bestehen immer wieder Unsicherheiten hinsichtlich der Datenspeicherung von Forschungsdaten, die oftmals in unterschiedlichen Formaten vorliegen.

\section{Unterschiedliche Forschungsinhalte und Anforderungen}

Wie unterscheidlich Fragestellungen zum FDM sein können, soll hier anhand von zwei Beispielen erläutert werden, eines aus der Bildungsforschung und der Linguistik sowie eines aus dem Bereich Software Systems Engineering (SSE).

Das interdisziplinäre Forschungsprojekt aus der Bildungsforschung und der Linguistik, ${ }^{18}$ beschäftigt sich damit, in welcher Form sich kulturelle Bildungsprozesse im Kontext von Digitalisierung verändern. Die Forschungsdaten stammen u. a. aus Portalen, Blogs sowie Plattformen im Internet. Es geht dabei um eine gemischtmethodische iterative, auf Massen- und Einzelfall gestützte Analyse der Digitalisierung von Rezensionsprozessen. Die Forschungsdaten, die im Projekt anfallen, sind heterogener Art mit quantitativen Korpusdaten und qualitativen Interviewdaten in Bild und Ton. Außerdem kommen selbsterzeugte Daten in MP3, txt, png, PDF und XML vor. Hier bezogen sich die Fragen auf die Erstellung eines DMP mit CLARIN Wizard DMTPY v.01, ${ }^{19}$ sowie die Aufbewahrung der Daten bei CLARIN-D. ${ }^{20}$ Weitere Hilfestellungen wurden zum Urheberrecht und zur Anonymisierung gewünscht.

Im zweiten Projekt haben Softwareentwickler im Bereich SSE ganz andere Anforderungen. Die Arbeitsgruppe Software Systems Engineering (SSE) ${ }^{21}$ arbeitet im Bereich Methoden und Techniken zur Unterstützung der effizienten Entwicklung von hoch-qualitativer Software, z.B. Software Produktlinienentwicklung, Requirements Engi-

18 https://www.empirische-bildungsforschung-bmbf.de/de/2510.ph $\mathrm{p}$ [29.9.2019].

19 https://www.clarin-d.net/de/aufbereiten/datenmanagementpla n-entwickeln [29.9.2019].

20 https://www.clarin-d.net/de/ [29.9.2019].

21 https://sse.uni-hildesheim.de/ [29.9.2019]. neering, Softwarearchitekturen, modelbasierter Entwicklung sowie Entwicklung adaptiver Systeme. Hier ging es nicht um die Erstellung eines DMP, sondern um die korrekte und dem Projektbedarf entsprechenden Speicherung der Datentypen und Datenformate. Dazu dienten lokale SVN-Repositorien im geschützten Universitätsnetzwerk, globale SVN-Repositorien im geschützten Netzwerk von Projektpartnern, öffentliche Git-Repositories, Youtube (für Videos) und lokale Festplatten auf den Mitarbeiter-Laptops und externen Festplatten (bei temporären bzw. Zwischenergebnissen). Auch wenn zunächst noch kein Datenmanagementplan entwickelt werden sollte, gab es den Wunsch nach einer eigenen disziplinspezifischen Leitlinie zum Umgang mit Forschungsdaten im Bereich SSE.

\section{Beratungsangebote}

Auf Empfehlung ${ }^{22}$ der Arbeitsgruppe Forschungsdaten der Schwerpunktinitiative „Digitale Information“ der Allianz der deutschen Wissenschaftsorganisationen ${ }^{23}$ wird für das Forschungsdatenmanagement von der UB Hildesheim jede mögliche Unterstützung erbracht. Die forschungsunterstützende FDM-Stelle versucht für alle Institute, Projekte und Forschende passgenaue Lösungen zu finden und sie „fit” für den Umgang mit Forschungsdaten zu machen, wie im Diskussionspapier „Research Data Vision 2025” der Arbeitsgruppe Forschungsdaten, der zweiten Phase der Schwerpunktinitiative „Digitale Information”, vorgeschlagen. ${ }^{24}$

Inzwischen gibt es beinahe täglich lokale Beratungen „on demand” für Forschende, ganze Forschungsprojekte, Graduiertenkollegs, Studierende und wissenschaftliche Mitarbeiterinnen und Mitarbeiter sowie Schulungsangebote in Form von Einzelberatungen (am Telefon, in der UB oder am Institut), Coffee Lectures und die fächerspezifischen Workshops.

$\mathrm{Zu}$ den generischen sowie den fachspezifischen Beratungen stehen jeweils Flyer, Poster und Handouts zur Verfügung. Bei externen Diensten wie CLARIN-D, GESIS, DIPF und Qualiservice wird bei Fragen zur Archivierung und Repositorien und weiterführenden fächerspezifischen Fragen Auskunft eingeholt, um nur einige Anbieter fachspe-

22 http://gfzpublic.gfz-potsdam.de/pubman/item/escido c:3007904:6/component/escidoc:3007905/Research_Data_Visio n_2025.pdf [15.9.2019].

23 https://www.dfg.de/dfg_profil/allianz/index.html [15.9.2019]. 24 http://gfzpublic.gfz-potsdam.de/pubman/item/escidoc: 3007904:6/component/escidoc:3007905/Research_Data_Vision_ 2025.pdf [15.9.2019]. 
zifischer Dienstleistungen zu nennen. Die meisten Fragen, lassen sich aber recht schnell vor Ort beantworten. Manchmal werden die Forschenden an externe Dienste vermittelt, wenn es sich um spezifische Details handelt. Die UB hat seit März 2018 einen guten Austausch mit den Fachbereichen und Instituten. Es gab zahlreiche Vorträge und Präsentationen bei der Forschungskommission, bei Fachbereichssitzungen und in Promovierendenseminaren, sowie eine Darstellung des Themas vor den Bibliotheksbeauftragten eines Fachbereichs.

Wissenschaftlerinnen und Wissenschaftler wünschen sich ganz unterschiedlich im Datenmanagement beraten und geschult zu werden. Gelernte Anwendungen wollen sie gleich für ihre aktuellen Projekte anwenden. Die UB bietet meist über E-Mail-Anfragen oder telefonische Beratung einen „First-Level Support” wie Daten bestmöglich gemanagt werden können. Meist kann dies sogleich erfolgen manchmal aber muss einige Tage recherchiert werden. Inhaltlich geht es häufig um das Speichern, die Rechtsthematik, Datenmanagementpläne, das Organisieren von Daten am Projektanfang sowie um Archivierungslösungen. Ebenso werden Ausküfte verlangt, welche Daten aufbewahrt werden müssen oder es wird um Einwilligungserklärungen und Muster für informierte Einwilligungen gebeten. Das Beratungsangebot wird in Zusammenarbeit mit den Forschenden aktiv weiterentwickelt und an die Erwartungen hinsichtlich forschungsunterstützender Dienste angepasst. Denn nachhaltiges FDM dient der wissenschaftlichen Integrität. Die Nachvollziehbarkeit des Entstehungsprozesses der Forschungsdaten (durch Generierung, Sekundärnutzung, Analyse, Speicherung, langfristige Sicherung und Dokumentation) garantiert deren Qualität und eröffnet gute Anschlussmöglichkeiten für die weitere Forschung. Die UB vermittelt hier, indem sie die Forschung betreut, und neben einer allgemeinen Informationskompetenz, nun auch die Forschungsdatenkompetenz fördert.

Studierende haben andere Fragen als Forschende. Bei ihnen bestehen eher Unsicherheiten zur Datenorganisation oder zur Nutzung von Cloud-Services wie der Academic Cloud $^{25}$ und zum mittelfristigen Speichern. Professorinnen und Professoren hingegen thematisieren die digitale Langzeitarchivierung und die Nachnutzung der gewonnenen Forschungsdaten. Für Datenmanagementpläne interessieren sich, nach den gemachten Beobachtungen, Studierende und Forschende, aber nicht aus jedem Fachbereich und Institut. Interesse am DMP besteht, wenn es um die Antragstellung geht, während für Studierende ein

25 https://academiccloud.de/home [15.9.2019].
DMP, z.B. mit RDMO, eher dazu dienen soll, die eigene Forschungsarbeit gut zu strukturieren.

Neben den Serviceangeboten gehört die Weiterentwicklung von Serviceinfrastrukturen und technischer Lösungen zu den wichtigsten Aufgaben. So wurde für das Erstellen von Datenmanagementplänen im März 2018 das Software-Werkzeug RDMO installiert und in Folge weiter an das fachspezifische Umfeld in den Instituten angepasst. Denn wenn ein Datenmanagementplan erstellt werden soll, ergeben sich diverse Fragen, die in einem Fragenkatalog in Untergruppen sortiert sind. Förderer wie die DFG geben oftmals eine Fragensammlung vor, die für ein Projekt beantwortet werden müssen. ${ }^{26}$

\section{Schulungen - Workshops - Coffee Lectures}

Fachhbereichs- und institutionsübergreifend steht, ganz besonders beim Verfassen von Projektanträgen, der Datenmanagementplan im Fokus. Daher wurde im Sommersemester 2019 ein dreistündiger Workshop zum Thema Datenmanagementpläne, inkl. RDMO, in Kooperation mit der Bergischen Universität Wuppertal durchgeführt. ${ }^{27} \mathrm{Da}-$ $\mathrm{zu}$ fanden ein RDMO- und ein Einführungs-Workshop für alle Fachbereiche statt. ${ }^{28}$

Allerdings sind längere Schulungen besonders für Forschende, die sich auf ihr Projekt konzentrieren wollen, ziemlich zeitraubend. So wurden als weiteres Format „Coffee Lectures“ eingführt, die als kleine Infohäppchen Wissen in kurzer Zeit vermitteln. Die ersten vier Coffee Lectures $^{29}$ behandelten die Grundlagen des Forschungsdatenmanagements, rechtliche Themen sowie Datenmanagementpläne und digitale Langzeitarchivierung. Unterstützt wurden diese von internen und externen Gästen mit Präsentationen aus ihrem Arbeitsbereich. Abgestimmt mit interessierten Forschenden werden weitere vier „Coffee Lectures“ zum Planen und Organisieren, Academic Cloud, Open Access und dem Umgang mit Metadaten stattfinden. ${ }^{30}$ Kaum nachgefragt wurden bisher Aspekte wie Metadaten, Ontologien sowie Semantic Web. Dazu

26 Folgende Fragenkataloge befinden sich aktuell auf der lokalen RDMO Instanz der SUH: RDMO, Ökonomie + DFG, Soziologie + DFG, Bildungswissenschaften + DFG, DFG.

27 https://zenodo.org/record/3234414\#.XZDjdXvgriU [29.9.2019]. 28 https://www.uni-hildesheim.de/forschungsdaten/?m=201901 [15.9.2019].

29 https://zenodo.org/record/2651654\#.XX6maXvgriU [15.9.2019]. 30 https://www.uni-hildesheim.de/forschungsdaten/ [15.9.2019]. 
werden Schulungen aus den Bereichen Sprach- und Informationswissenschaften, Erziehungs- und Sozialwissenschaften, Kulturwissenschaften, Informatik und verschiedene Use-Cases aus der Forschung angeboten. Bisher wurden diese von den Fachbereichen sehr gut angenommen.

\section{Fazit und Ausblick}

Mit der Einrichtung einer dauerhaften Stelle für das Forschungsdatenmanagement wurde dieses Thema fest an der SUH installiert. Es können nun jederzeit Fragen zur Datensicherung, Datenorganisation, Dokumentation sowie zu rechtlichen und ethischen Anforderungen gestellt und beantwortet werden. Dies gilt sowohl für allgemeine wie auch disziplinspezifische Ansätze. Im Bereich der Beratungs- und Schulungsangebote bereitet die UB Hildesheim weiterhin neue Muster-Workflows und Muster-Einwilligungen (Survey-Daten, Interviewdaten und Nutzungsrechte) vor, da diese häufig nachgefragt werden und es noch an geeigneten Materialien fehlt.

Ebenso wichtig ist eine verstärkte Zusammenarbeit mit den einzelnen Instituten. Hier gilt es den Speicherbedarf der institutionellen Forschungsdaten-Repositorien besser abzuschätzen. Handlungsbedarf besteht außerdem beim Aufbau von weiteren lokalen Unterstützungsangeboten. So wäre es für die Fachbereiche und einzelne Institute hilfreich „Data Stewards“ zu benennen. Diese unterstützen den Umgang mit Daten und helfen, die technischen Infrastrukturen zu verbessern und weiter zu entwickeln. Dazu wäre es sinnvoll eigene institutionelle ForschungsdatenLeitlinien zur Orientierung zu verfassen, die auf disziplinspezifische Angebote hinweisen, wie dies z.B. am Institut für Informatik, in der AG Software Systems Engineering, diskutiert wurde. Auch müssen noch weitere fachspezifische Fragenkataloge für RDMO und Kataloge für andere Forschungsförderer entwickelt werden.

Die Universität Hildesheim enagagiert sich aktiv auf dem dynamischen Entwicklungsgebiet des Forschungsdatenmanagement. Ein Anfang zur Sensibilisierung wurde durch erste Schulungsmaßnahmen gemacht und gut angenommen. Das Aufgabenfeld wird für alle Institute und Fachbereiche zukunftsgerichtet bleiben. Dabei wird die FDM-Stelle in der UB der „First-Point-of-Contact” bleiben und weiterhin fächerspezifisch und fachübergreifend tätig sein. Die Schulungen werden nach und nach mehr in die Tiefe gehen. Die UB nimmt ihre Rolle bei der Vermittlung von Informationskompetenzen ernst. Dabei stellt das FDM eine neue Facette dieses Themenkomplexes dar. Mit „Data Literacy” als Teil des FDM hat die UB einen neuen
Bereich in einer wissenschaftlichen Bibliothek verankert. Sinnvoll wären in naher Zukunft die Einrichtung von „Digital Research Labs” (Gestaltungslabore) sowie „Library Carpentries" (Makerspaces).

Deskriptoren: Forschungsdaten, Forschungsdatenmanagement, Universitätsbibliothek, Bibliothek, Digitale Forschungsinfrastruktur, Schulungen, Workshops, Coffee Lectures, Infrastrukturen

\section{Literatur}

Deutsche Forschungsgemeinschaft (2019). Umgang mit Forschungsdaten. URL: http://www.dfg.de/foerderung/antrag_gutachter_ gremien/antragstellende/nachnutzung_forschungsdaten/ [15.9.2019].

Pinfield, S., Cox, A. M., \& Rutter, S. (2017). Mapping the future of academic libraries: a report for SCONUL. URL: https://sconul.ac. uk/publication/mapping-the-future-of-academic-libraries [16.9.2019].

Strauch, A. (2019): Coffee Lectures presented by your library. Forschungsdatenmanagement an der Universität Hildesheim. Unterstützung durch die UB. DOI: https://DOI10.5281/zeno do.2651654 [15.9.2019].

Whyte, A., Tedds, J. (2011). 'Making the Case for Research Data Management'. DCC Briefing Papers. Edinburgh: Digital Curation Centre. URL: http://www.dcc.ac.uk/resources/briefing-papers [15.9.2019].

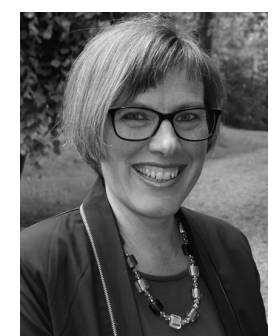

Annette Strauch, M. A.

Universitätsbibliothek Hildesheim

Forschungsdatenmanagement

Universitätsplatz 1

31141 Hildesheim

annette.strauch@uni-hildesheim.de

Annette Strauch arbeitet seit 2012 im Bereich Forschungsdatenmanagement und Digitale Langzeitarchivierung (kiz, Universität Ulm, ZIMT, Universität Siegen, SFB 1187, INF-Projekt). In der Universitätsbibliothek Hildesheim ist sie seit März 2018 für alle Bereiche im Forschungsdatenmanagement die Ansprechperson. Im Vordergrund des Forschungsdatenmanagement stehen für sie die Nutzerinnen und Nutzer der technischen und serviceorientierten Infrastrukturen sowie der Austausch mit den Akteurinnen und Akteuren in der globalen Forschungsdaten-Community. 